\title{
Enzymatic Crosslinking of Polymer Conjugates is Superior over Ionic or UV Crosslinking for the On-Chip Production of Cell-Laden Microgels
}

\author{
Sieger Henke, Jeroen Leijten, Evelien Kemna, Martin Neubauer, \\ Andreas Fery, Albert van den Berg, Aart van Apeldoorn, Marcel Karperien*
}

Cell-laden micrometer-sized hydrogels (microgels) hold great promise for improving high throughput ex-vivo drug screening and engineering biomimetic tissues. Microfluidics is a powerful tool to produce microgels. However, only a limited amount of biomaterials have been reported to be compatible with on-chip microgel formation. Moreover, these biomaterials are often associated with mechanical instability, cytotoxicity, and cellular senescence. To resolve this challenge, dextran-tyramine has been explored as a novel biomaterial for on-chip microgel formation. In particular, dextran-tyramine is compared with two commonly used biomaterials, namely, polyethylene-glycol diacrylate (PEGDA) and alginate, which crosslink through enzymatic reaction, UV polymerization, and ionic interaction, respectively. Human mesenchymal stem cells (hMSCs) encapsulated in dextran-tyramine microgels demonstrate significantly higher (95\%) survival as compared to alginate (81\%) and PEGDA (69\%). Long-term cell cultures demonstrate that $\mathrm{hMSC}$ in PEGDA microgels become senescent after $7 \mathrm{~d}$. Alginate microgels dissolve within $7 \mathrm{~d}$ due to $\mathrm{Ca}^{2+}$ loss. In contrast, dextran-tyramine based microgels remain stable, sustain hMSCs metabolic activity, and permit for single-cell level analysis for at least $28 \mathrm{~d}$ of culture. In conclusion, enzymatically crosslinking dextran-tyramine conjugates represent a novel biomaterial class for the on-chip production of cell-laden microgels, which possesses unique advantages as compared to the commonly used UV and ionic crosslinking biomaterials.

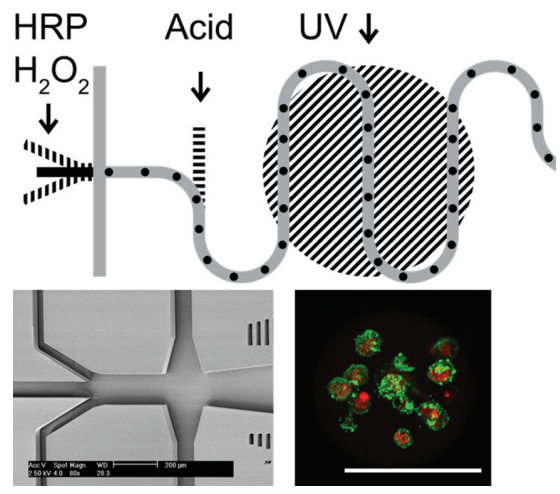

S. Henke, Dr. J. Leijten, Dr. A. van Apeldoorn, Prof. M. Karperien Department of Developmental BioEngineering

MIRA Institute for Biomedical Technology

and Technical Medicine

University of Twente

P.O. Box 217

7500AE Enschede, The Netherlands

E-mail: h.b.j.karperien@utwente.nl

Dr. E. Kemna, Prof. A. van den Berg

BIOS Lab on a Chip group

MESA + Institute for Nanotechnology

University of Twente

P.O. Box 217

7500AE Enschede, The Netherlands
Dr. M. Neubauer

Department of Physical Chemistry II

University of Bayreuth

Universitätsstrasse 30, 95447 Bayreuth, Germany

Prof. A. Fery

Leibniz Institut für Polymerforschung Dresden e.V.

(Leibniz Institute of Polymer Research Dresden)

Institute of Physical Chemistry and Polymer Physics

Hohe Str. 6, 1079 Dresden, Germany

Prof. A. Fery

Chair of Physical Chemistry of Polymeric Materials

Technische Universität Dresden

1079 Dresden, Germany 


\section{Introduction}

The cellular microenvironment strongly controls the function and fate of cells. Consequently, removing cells from their natural environment drastically and progressively alters a cell's phenotype and capacity to perform its native function. ${ }^{[1-3]}$ Encapsulating cells in engineered biomimetic environments using instructive biomaterials therefore represents a powerful tool to control cell behavior. ${ }^{[4,5]}$ One of the most common methods for embedding cells in a 3D environment is through hydrogels encapsulation. ${ }^{6]}$ Hydrogel design parameters currently are focused on balancing the biomaterial's physical properties including, e.g., shape, stiffness, porosity, and degradation rate and chemical cues including presentation of specific cues such as matrix and growth factors. However, the demands in hydrogel design from hydrogel-host perspective and hydrogel-encapsulated cells perspective are often conflicting. This can be resolved by microencapsulating cells first in an instructive micrometer-sized biomaterial, which in turn is incorporated in a second biomaterial with distinct properties.

Unfortunately, only a limited amount of biomaterials have been explored for microencapsulation. ${ }^{[7-11]}$ These biomaterials are typically associated with poor mechanical properties, significant amounts of cell death, and insufficient levels of cell stimulation. This has resulted in suboptimal outcomes in short term culture results and incompatibility with long term cultures.

Of the explored materials, alginate has received a substantial amount of attention. ${ }^{[12]}$ Alginate is a polysaccharide extracted from the cell walls of brown algae that is typically crosslinked using divalent ions such as $\mathrm{Ca}^{2+}$. However, $\mathrm{Ca}^{2+}$ is lost over time due to diffusion, leading to a progressive uncontrolled decrease in stiffness and inevitable structural failure of the microgel. ${ }^{[13]}$ Another commonly explored biomaterial for microfluidic microgel formation is the synthetic polymer polyethylene-glycol $(\mathrm{PEG}){ }^{[8]}$ Fast, on-chip crosslinking of PEG microgels requires photo crosslinking, e.g., PEG diacrylate (PEGDA), as other crosslinking methods like thiol-ene click chemistry take too much time to crosslink on-chip only. ${ }^{[14]}$ However, this requires high power UV irradiation, which is correlated with suboptimal cell survival and potential genetic damage. ${ }^{[15]}$ Next to these, several other less commonly explored biomaterials have been reported for the production of cell-laden microgels, such as thiol conjugated hyaluronic acid. ${ }^{[14]}$ However, these materials have crosslinking times ranging from minutes to hours. This requires off chip crosslinking, for which droplets need to be stabilized by powerful surfactants. ${ }^{[14,16]}$ These approaches demand additional time consuming steps to be compatible with down-stream processes. Thus, there is a clear need for an on-chip crosslinking biomaterial that allows for high cell survival, does not induces genetic damage, and allows for facile chemical modification to allow the fabrication of biomimetic microenvironments.

Here, we report on dextran-tyramine conjugates (DexTA) as a novel biomaterial for microencapsulation. ${ }^{[17]}$ Dextran is a natural polymer with excellent biocompatibility. Once conjugated to tyramine, the resulting polymer conjugate can be enzymatically crosslinked using horseradish peroxidase in the presence of hydrogen peroxide. It is potentially ideal for on-chip microgel formation, due to its extremely fast, yet cell friendly gelation. Moreover, numerous modifications are available to tune Dex-TA's bioactivity, degradability, and mechanical stiffness. ${ }^{[18,19]}$

In order to make a direct comparison between photo, ionic, and enzymatic crosslinking, we developed a novel universal microfluidic chip. This device allows for the controlled crosslinking of each of the three mentioned methods. Using this platform, we have compared microencapsulation of human mesenchymal stem cells (hMSCs) in PEGDA, alginate and Dex-TA microgels, in long-term cell cultures. In short, we have shown that on-chip enzymatically crosslinking of cell-laden microgels has superior properties in terms of cell survival, metabolic activity, and stability in both short and long-term cell cultures, as compared to ionically crosslinked alginate and UV crosslinked PEGDA.

\section{Results}

\subsection{Droplet Formation and Characterization}

Using the universal chip design (Figure 1), we determined the stable droplet formation regime for each polymer. Stable monodispersed droplets were formed in a size range of $137( \pm 2.7)-201( \pm 5.3) \mu \mathrm{m}, 127( \pm 1.9)-142( \pm 6.5) \mu \mathrm{m}$, and $122( \pm 1.6)-168( \pm 8.3) \mu \mathrm{m}$ for PEGDA, Alginate and Dex-TA solutions, respectively (Figure 2). In subsequent experiments we used a flow ratio of the dispersed/continuous phase of 0.14 for Dex-TA and PEGDA, resulting in droplets of respectively $157( \pm 3.2)$ and $174( \pm 5.0) \mu \mathrm{m}$ in diameter. At this flow rate, PEGDA containing droplets are exposed to UV-light for approximately $8 \mathrm{~s}$. To achieve reasonably stable crosslinking into a microgel within this time frame a minimal UV light intensity of $140 \mathrm{~mW} \mathrm{~cm}{ }^{-2}$ was required. Lower UV light intensity or higher flow speed resulted in microgels that disintegrated within a few hours of culture indicative for incomplete crosslinking (data not shown). Higher UV intensity resulted in better crosslinking but this proved even more detrimental for survival of the encapsulated cells as explained in more detail below. The maximum achievable stable flow rate ratio for alginate was noticeably lower (0.075) resulting in droplets of $142( \pm 5.7) \mu \mathrm{m}$. The possible size range of the droplets using

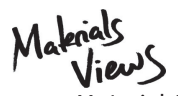

www.MaterialsViews.com 

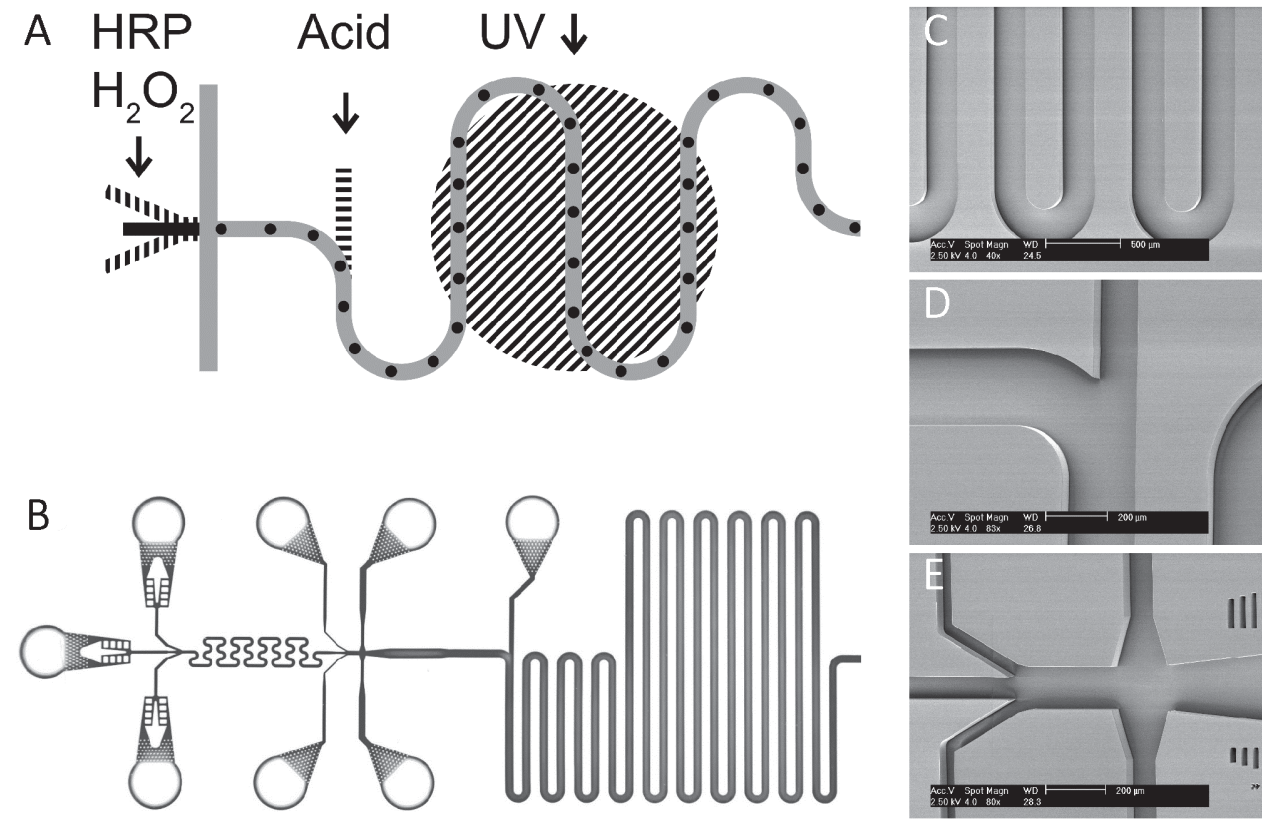

Figure 1. A) Schematic overview of the universal microfluidic chip used for production of photo crosslinked PEGDA, ionic crosslinked alginate, and enzymatically crosslinked Dex-TA microgels. Dashed areas indicate where photo, ionic, and enzymatic crosslinking are initiated. B) Photograph of the universal microfluidic chip containing a black dye to visualize inlets on the left, incubation channel and outlet on the right. Scanning electron images were made of $C$ ) the incubation channel that is UV irradiated to crosslink PEGDA, D) the addition channel to add acidified oil to crosslink alginate, and $\mathrm{E}$ ) the droplet forming nozzle with inlets for HRP and $\mathrm{H}_{2} \mathrm{O}_{2}$ to crosslink Dex-TA. Scale bars depict (C) $500 \mu \mathrm{m}$ or (D,E) $200 \mu \mathrm{m}$.

alginate was restricted, due to the highly viscous nature of the $2 \%$ alginate solution.

\subsection{Microgel Formation}

Droplets of hydrogel precursors with or without cells were produced by emulsification, followed by on-chip crosslinking of the cell-laden gel precursor droplets using UV irradiation, exposure to bivalent $\mathrm{Ca}^{2+}$ ions, or enzymatic reaction. Crosslinked microgels were post-chip collected in culture medium. The emulsion was broken by washing with culture medium and the microgels were collected in the aqueous phase. The fabricated microgels were monodisperse within a narrow size distribution (Figure $3 \mathrm{~A}-\mathrm{C}$ ). The microgels of Dex-TA were slightly opaque, and have some patches on the surface caused by the rapid initiation of crosslinking (Figure 3C). The microgel shape was determined by the channel geometry and the droplet's volume. In particular, microgels were spheres with a flat top and bottom. Scanning electron microscopy (SEM) preparation required dehydration of the microgels, which induced microgel shrinkage. SEM analysis confirmed the shape and uniformity of the microgels, particularly of the PEGDA and alginate microgels (Figure 3D-F). The surface of the

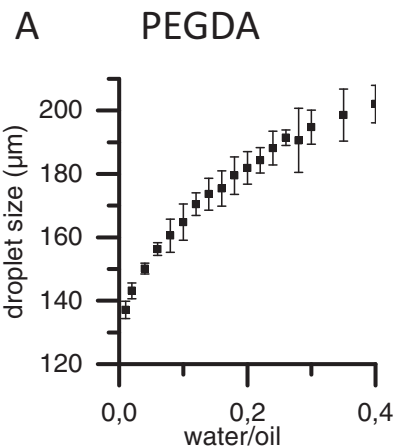

B Alginate

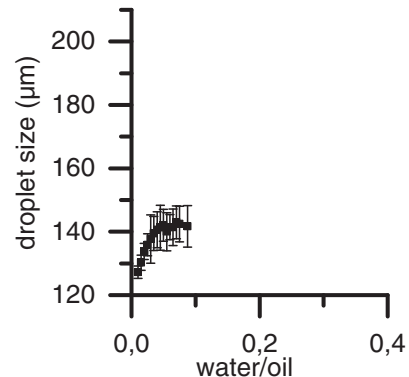

C Dex-TA

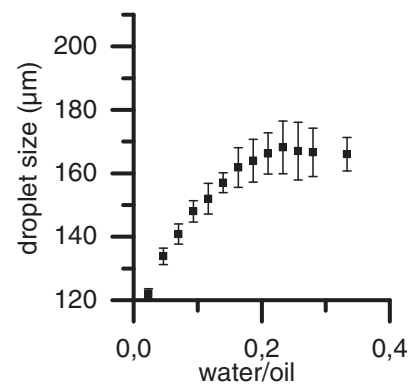

Figure 2. PEGDA, alginate, and Dex-TA solutions were infused in the universal chip and droplet sizes were measured on-chip using serial microphotography. Graphs show the average droplet diameter at varying flow speed ratios of A) PEGDA, B) alginate, and C) Dex-TA. 

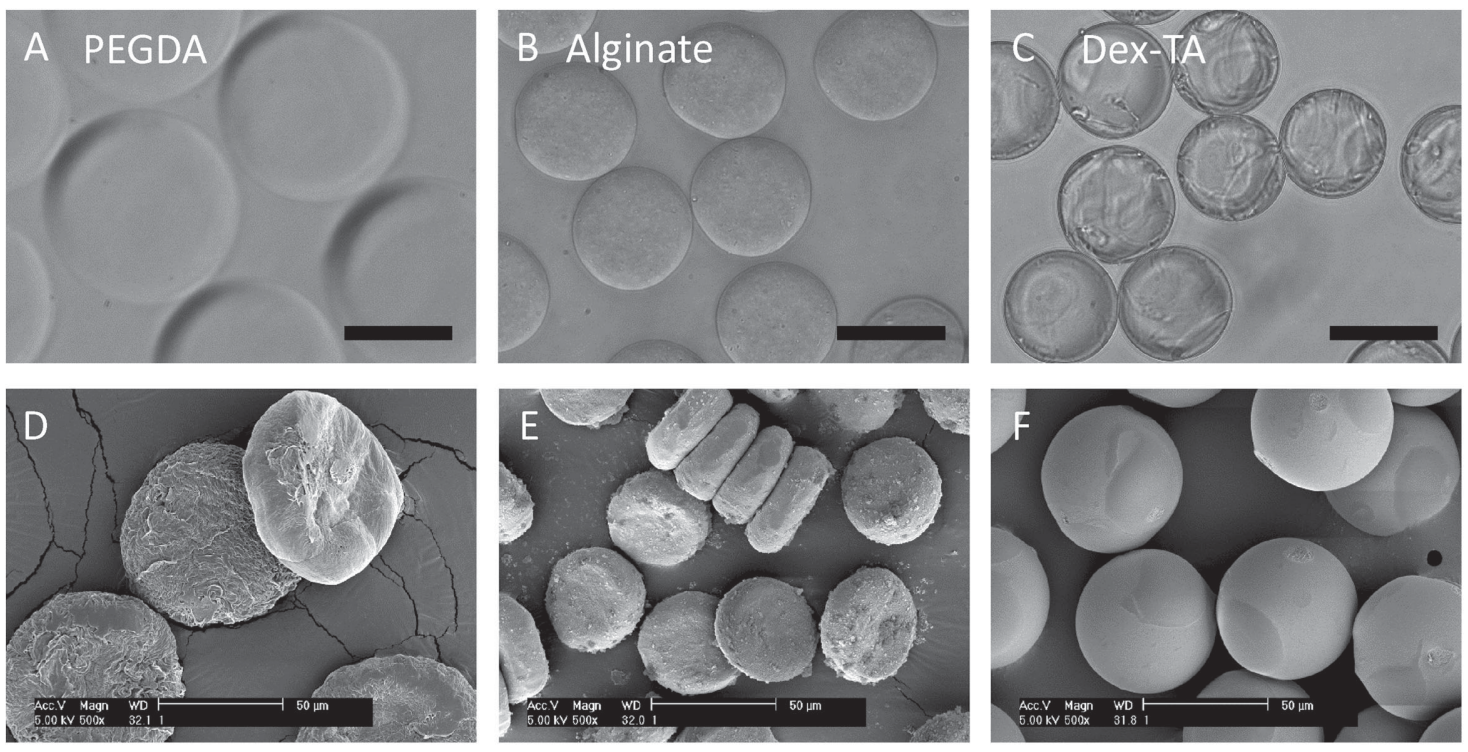

Figure 3. Microgels were microphotographed post-collection using $A-C)$ brightfield microscopy and D-F) SEM. Microgels were composed of $(A, D)$ UV crosslinked PEGDA, $(B, E)$ ionically crosslinked alginate, and (C,F) enzymatically crosslinked Dex-TA. Scale bars of (A-C) depict $100 \mu \mathrm{m}$ and (D-F) depict $50 \mu \mathrm{m}$.

PEGDA microgels appeared somewhat irregular which is likely an artefact of the dehydration process and incomplete crosslinking (Figure 3D). Alginate gels have some salt residues on them, which precipitated during dehydration. The Dex-TA microgels were smooth, with two patches (Figure 3F). These patches likely originated from the instantaneous initiation of the crosslinking of the polymer conjugates at the inlets of $\mathrm{HRP}$ and $\mathrm{H}_{2} \mathrm{O}_{2}$ just before droplet formation (Figure 1E).

\subsection{Mechanical Properties}

The E-modulus of the microgels was measured using atomic force microscopy (Figure 4). UV crosslinked PEGDA microgels proved relatively weak, with an E-modulus of

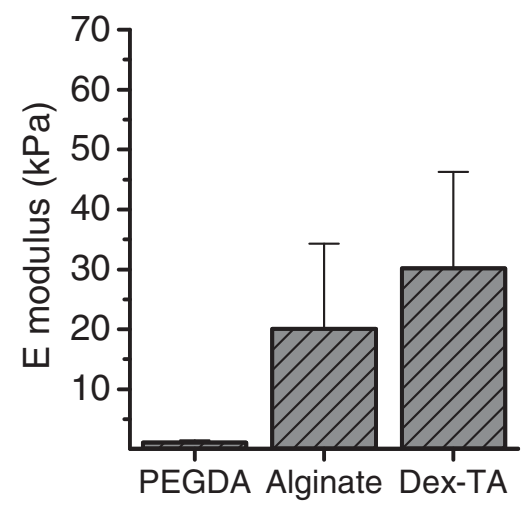

Figure 4. E-moduli of 10\% PEGDA, $2 \%$ alginate, and 10\% Dex-TA microgels as measured by AFM.
$1.1 \mathrm{kPa}$, which is considerably lower than values reported in literature of a fully cross linked PEGDA hydrogel of similar composition and wt/vol\%, indicative for incomplete crosslinking. ${ }^{[20]}$ Moreover, ionically crosslinked alginate microgels were significantly stronger with an E-modulus of to $20.1 \mathrm{kPa}$, and the enzymatically crosslinked Dex-TA microgels demonstrated the highest E-modulus at $30.2 \mathrm{kPa}$.

\subsection{Cell Survival}

We then investigated the survival of hMSCs microencapsulated in PEGDA, alginate and Dex-TA microgels (Figure 5A). For PEGDA microgels, the survival of hMSCs proved inversely correlated with the UV-dose (Figure 5B). Maximal cell survival was $69 \%$ at $140 \mathrm{~mW} \mathrm{~cm}^{-2} \mathrm{UV}$ irradiation. Usage of lower UV intensity, potentially leading to higher cell survival, was insufficient to crosslink the PEGDA microgels on-chip, as these microgels dissolved within hours of culture (data not shown). For alginate, cell survival correlated with the acetic acid concentration within the oil, which was required to release bivalent $\mathrm{Ca}^{2+}$ ions from the insoluble $\mathrm{CaCO}_{3}$ nanoparticles (Figure 5B). The maximal cell survival for alginate was $81 \%$, which was achieved at an acetic acid concentration of $0.44 \mu \mathrm{L} \mathrm{mL} \mathrm{L}^{-1}$ oil. Lower acetic acid concentrations resulted in the fusion of microgels. In contrast, enzymatic crosslinking resulted in significantly lower cell death, with cell survival rates of $>95 \%$ in a wide range of $\mathrm{H}_{2} \mathrm{O}_{2}$ concentrations (Figure $5 \mathrm{C}$ ). As theoretically every crosslink requires a single $\mathrm{H}_{2} \mathrm{O}_{2}$ molecule, increasing the concentration of $\mathrm{H}_{2} \mathrm{O}_{2}$ increases

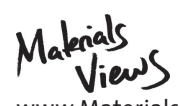

www.MaterialsViews.com 
A

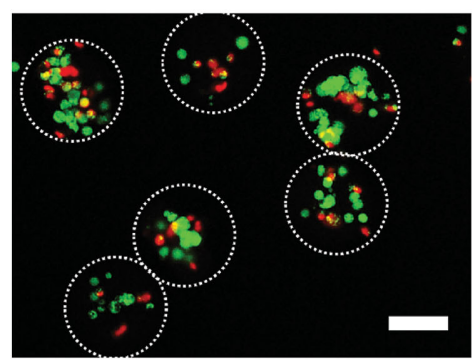

D

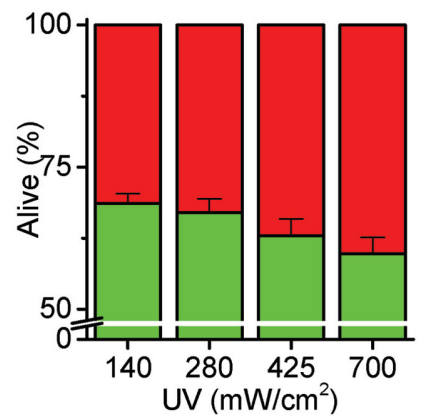

B

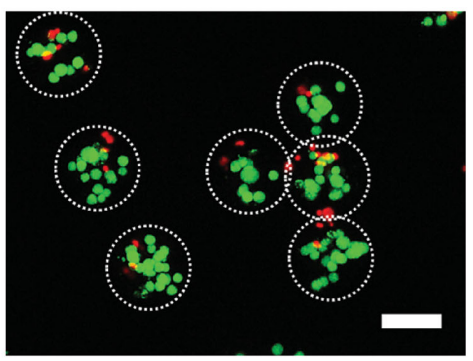

E

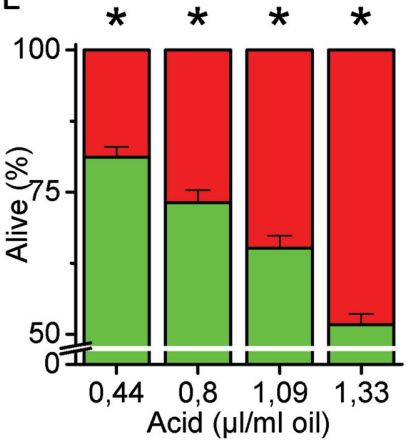

C Dex-TA

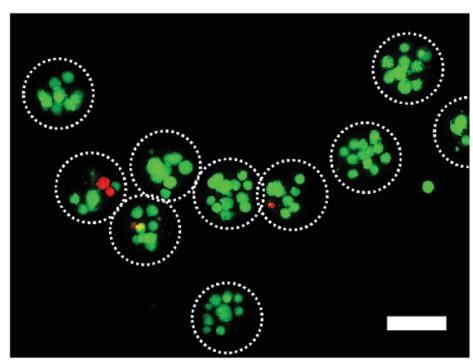

$\mathrm{F}$

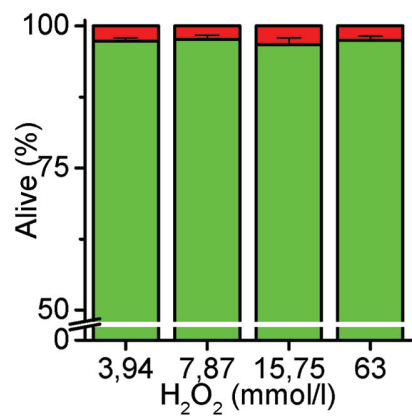

Figure 5. hMSC laden microgels stained for cell survival (green) and cell death (red). A) PEGDA exposed to $140 \mathrm{~mW} \mathrm{~cm}^{-2}$ of UV. B) Alginate exposed to $0.44 \mu \mathrm{L} \mathrm{mL}^{-1}$ of acetic acid. C) Dex-TA exposed to $15.75 \mathrm{mmol} \mathrm{L}^{-1}$ of $\mathrm{H}_{2} \mathrm{O}_{2}$. Scale bars depict $100 \mu \mathrm{m}$. D-F) Semiquantification cell survival based on live/dead microphotographs of (D) PEGDA, (E) alginate, and (F) Dex-TA. Error bars represent standard error of the mean. * represents a p-value of $<0.05$ compared to the other conditions in the material.

the total amount of crosslinks. However, a too high concentration of $\mathrm{H}_{2} \mathrm{O}_{2}$ inhibits the function of HRP, thus eventually leading to a reduction in crosslinking rate (data not shown). ${ }^{[21]}$

\subsection{Metabolic Activity}

We then microencapsulated hMSCs in Dex-TA, alginate, and PEGDA microgels, and subsequently cultured the microgels for four weeks. At predefined regular intervals, the metabolic activity of the encapsulated cells was determined using a presto blue assay. In PEGDA the metabolic activity of hMSCs dropped to undetectable levels within $7 \mathrm{~d}$ post-encapsulation (Figure $6 \mathrm{E}$ ). In contrast, hMSCs microencapsulated in alginate remained metabolically active up to at least $7 \mathrm{~d}$ (Figure 6F). However, $7 \mathrm{~d}$ post-encapsulation the microgels disintegrated, which was likely due to the ion exchange with the culture media. This undermined the ability to reliably measure the encapsulated cells' metabolic activity after day 7. In marked contrast, Dex-TA microgels remained stable over the whole culture period, and the encapsulated cells continued to be metabolically active for at least $28 \mathrm{~d}$. Importantly, after a small initial drop in metabolic activity at day 4, the metabolic activity progressively increased over time (Figure 6G). The metabolic data was corroborated using an MTT assay at day 7 (Figure 6D, Figure S1, Supporting Information).

\section{Discussion and Conclusions}

In this paper we have presented a microfluidic platform that was used for on-chip encapsulation of cells in stable microgels in a size range of $120-200 \mu \mathrm{m}$. This device allowed for the universal crosslinking of different types of water soluble polymers that are based on an ionic, UV- or enzymatic initiation. Most importantly, we explored enzymatically crosslinking tyramine conjugated polymers as novel materials for microencapsulation, using Dex-TA as a prototype material. Dex-TA macrogels haven been demonstrated to possess excellent properties for cell encapsulation and biofunctionality. ${ }^{[17,22,23]}$ Here, we compared enzymatically crosslinked Dex-TA microgels with photo crosslinked PEGDA and ionically crosslinked alginate, using an identical microfluidic droplet generator design.

The on-chip UV based crosslinking of PEGDA demonstrated major drawbacks caused by the limited time window for UV irradiation due to the relatively short onchip residence time. This therefore demanded high intensity UV exposure to ensure on-chip microgel curing, which caused significant levels of cytotoxicity, as also previously described in literature. ${ }^{[24]}$ Surprisingly, PEGDA microgels proved to be the mechanically weakest gels in our comparison, and demonstrated limited cell survival, even after minimizing the UV intensity. Although these weak gels could be suited for applications that require a matrix 
A PEGDA

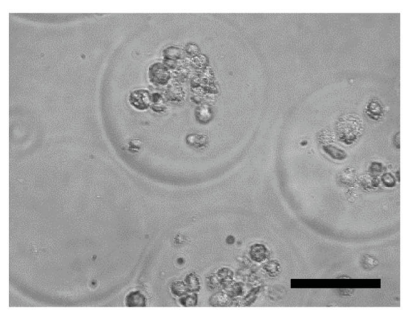

$\mathrm{E}=$

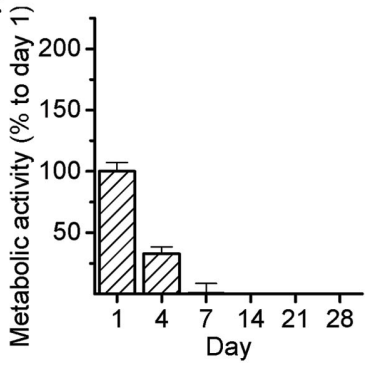

B Alginate

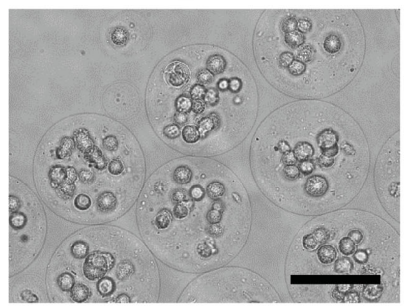

$\mathrm{F}$

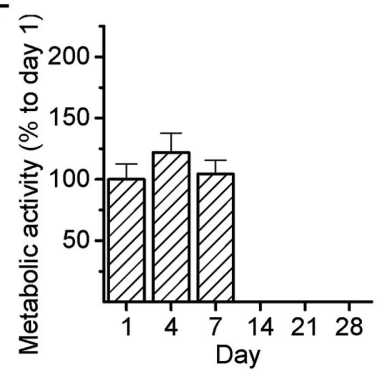

C Dex-TA

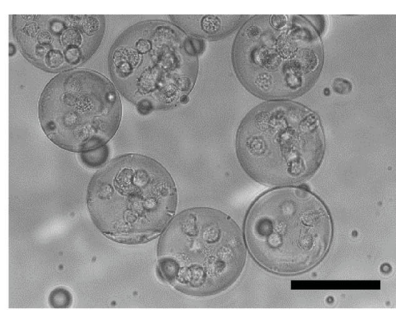

$G_{F}$

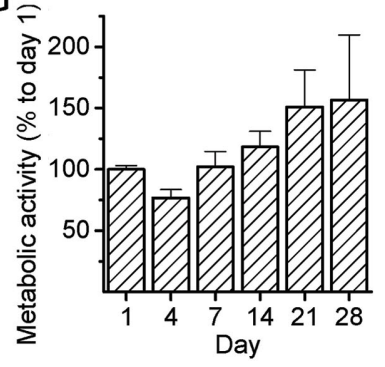

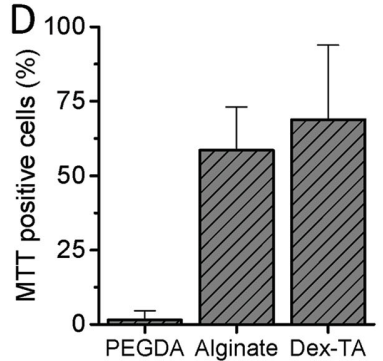

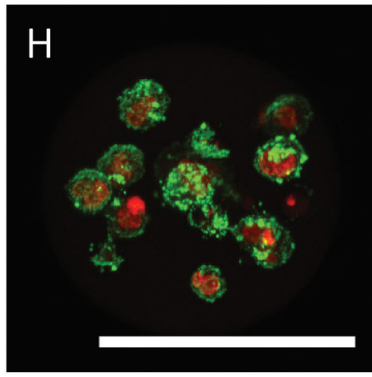

Figure 6. Brightfield microphotographs of hMSCs encapsulated in A) PEGDA, B) alginate, and C) Dex-TA microgels at day 1 post-crosslinking. D) MTT quantification of microgels after $7 \mathrm{~d}$ of culture $(n>10)$. Metabolic activity of hMSCs encapsulated in E) PEGDA, F) alginate, and G) Dex-TA as measured by prestoblue assay on day 1, 4, 714, 21, and 28 and normalized to day 1. H) Z-stacked confocal laser scanning microscopy image of the cytoskeleton (green) and nucleus (red) of hMSCs encapsulated in a Dex-TA microgel after $14 \mathrm{~d}$ of culture. Scale bar depicts $100 \mu \mathrm{m}$.

stiffness in the order of magnitude of $1 \mathrm{kPa}$, e.g., for cells of neurogenic lineage, it also limited their ease of handling due to their fragile nature. ${ }^{[25]}$ It is expected that further increasing the UV intensity or photoinitiator concentration would produce stronger gels due to a higher density of crosslinks, but would likely also further lower the cell survival. Indeed by increasing the UV intensity from $140 \mathrm{~mW} \mathrm{~cm}{ }^{-2}$, which was minimally required to obtain a more or less stable microgel configuration in the $8 \mathrm{~s}$ of retention time in the incubation channel to $700 \mathrm{~mW} \mathrm{~cm}^{-2}$, decreased the already low cell survival even further. At the UV intensity of $140 \mathrm{~mW} \mathrm{~cm}{ }^{-2}$ crosslinking was still incomplete as demonstrated by rheology. We obtained gels with an E-modulus of approximately $1.1 \mathrm{kPa}$ which is considerably lower compared to the E-modulus of a fully crosslinked 10\% wt/v PEGDA hydrogel having an E-modulus larger than $10 \mathrm{kPa}{ }^{[20]}$ Phototoxicity could potentially be mitigated through flow speed and UV reduction, which would also limit the production speed. Alternatively, the microgel's on-chip residence time could be enhanced via on-chip delay lines, which would result in suboptimal chip designs, because of high pressure buildup and thus increased chance of chip failure, that still associate with substantial amounts of cell death. In addition, on-chip UV based crosslinking of PEGDA microgels induced metabolic cell senescence in the surviving cells within a week, which potentially indicates extensive cell damage. UV induced cell death and damage could also be minimized by crosslinking the microgels off-chip at low intensity for a prolonged period of time. ${ }^{[14]}$ Indeed, photocrosslinking of PEGDA into stable cell laden hydrogels is commonly achieved by crosslinking for at least $5 \mathrm{~min}$ at relatively low UV intensity. ${ }^{[20,26,27]}$ However, this approach would require long retention of droplets off-chip in stable emulsions, which has the adverse effect of demanding additional complex and time consuming procedures. In particular, powerful surfactants will be required for the off chip stabilization of cell encapsulating droplets, which are notoriously difficult to remove. From this work PEGDA appears not very well suited for the on-chip crosslinking polymers into cytocompatible and stable microgels.

Ionic crosslinking of alginate is commonly achieved by dropping alginate in a solution with divalent metal ions such as $\mathrm{Ca}^{2+}$. However, this gelation method, which crosslinks from the outside in, has been found unsuitable for the on-chip fabrication of smaller alginate microgels due to the formation of nonuniform and inhomogeneous microgels. ${ }^{[28]}$ Instead, an internal gelation method is required, which is based on solubilizing calcium carbonate particles in the polymer precursor by a temporary decrease in the droplet's $\mathrm{pH}$, using an oil and water soluble acid. ${ }^{[28]}$ Unfortunately, this decrease in $\mathrm{pH}$ is toxic to the encapsulated cells. We indeed observed a direct correlation between acid concentration and cell survival, with a maximum survival of $81 \%$, as also found by Tan and Takeuchi. ${ }^{[28]}$ Furthermore, it is likely that the surviving cells experience substantial amounts of acid induced stress. Another potential disadvantage of this system is the rapid deterioration of the microgels due to progressive loss of calcium ions during culture. ${ }^{[29]}$ Deterioration of the microgels progressed on a strikingly higher pace than those reported for macrosized hydrogels, likely due to the

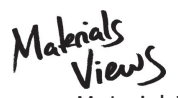

www.MaterialsViews.com 
vastly increased surface-to-volume ratio. For short term cultures such as toxicology studies, this fast deterioration is not likely to pose a limitation. However, it does present a challenge for long-term cultures such as cell differentiation, which usually takes over two weeks. In vitro the microgels can be stabilized by additional supplementations of $\mathrm{Ca}^{2+}$ ions, which might affect cell behavior. Moreover, a repetitive crosslinking approach is not compatible with in vivo applications in a facile manner.

In contrast to these ionic and UV based crosslinking approaches, our body primarily relies on enzymatic induced crosslinking. Examples of enzymes involved in the crosslinking of extracellular matrix molecules are transglutaminase, lysyl oxidase, and horseradish peroxidase. ${ }^{[30-32]}$ A major advantage of this approach is its ability to crosslink at physiological conditions, without requiring harsh chemicals. In this study, we explored microgel formation using the enzymatic crosslinking of Dex-TA, which was achieved by on-chip mixing of polymer precursor with the enzyme $\mathrm{HRP}$ and $\mathrm{H}_{2} \mathrm{O}_{2}$. In contrast to UV or acid induced systems, enzymatic crosslinking strategies are well-known for their cytocompatibility. Although it might be intuitive to reason that the use of $\mathrm{H}_{2} \mathrm{O}_{2}$ might induce cytotoxic events, the required levels of $\mathrm{H}_{2} \mathrm{O}_{2}$ are far below those that induce cell damage or death, as the minute quantities of $\mathrm{H}_{2} \mathrm{O}_{2}$ are rapidly converted during the enzymatic reaction. Indeed, cell survival after microgel formation was substantially higher as compared to other reported microgel crosslinking strategies, reaching levels as high as 95\%. In fact, the observed level of cell death was identical to those generated by routine cell passaging. The cell-friendliness of this approach was further underlined by the observation that the cell survival was not adversely affected by increasing the $\mathrm{H}_{2} \mathrm{O}_{2}$ concentrations to levels that exceeded the functional crosslinking concentration. Dex-TA microgels of $10 \%$ Dextran were with $30 \mathrm{kPa}$ mechanically very strong. This makes Dex-TA microgels very suitable for differentiation toward the musculoskeletal lineage, where cartilage needs $20-30 \mathrm{kPa}$ and bone $25-40 \mathrm{kPa} \cdot{ }^{[25,33]} \mathrm{How}-$ ever, other tissues like nerve or brain $(0.1-1 \mathrm{kPa})$ and fat (3 $\mathrm{kPa}$ ) require softer gels. ${ }^{[25,33]}$ In order to match these, the mechanical properties can be tuned by varying the wt/vol\% of the gel and the degree of substitution of the dextran. ${ }^{[17,22]}$

Encapsulating cells in micromaterials that in turn will be encapsulated in a macrosized scaffold opens up numerous potential avenues in creating multiscale bioengineered constructs. In particular, it is conducive for the decoupling of the biomaterial that is proximal to the cells and the bulk biomaterial that is more distal to cells. ${ }^{[34]}$ This strategy resembles the natural organization of cells in tissue, in which cells are surrounded by a pericellular matrix, which together is embedded in an extracellular matrix. The composition and thus function differs greatly between these matrices. Our strategy provides a roadmap toward engineering such complex and biologically relevant organizations. This approach holds great potential to advance tissue engineering by adding biological complexity in a facile manner to the tissue engineered constructs. ${ }^{[35-37]}$ This development could additionally contribute to other fields such as (stem)cell biology, drug development, and pathophysiology, by driving the development of more biomimetic ex vivo models. ${ }^{\text {[38-40] }}$ Moreover, gaining the capability to create cell-friendly customizable cellular microniches provides possibilities for efficient high throughput screens with single-cell resolution. ${ }^{[41]}$ This might be of particular interest as microgels can yield information on the single cell level by confocal microscopic analysis of the entire gel, and allow for facile downstream analysis. ${ }^{[41]}$ This approach allows for the precise probing of cellular heterogeneity to a given biophysical, chemical or environmental stimuli to identify of, e.g., differently responsive sub-populations of cells. Moreover, these 3D biomimetic microtissues can act as smart building blocks to create complex functional tissues following a bottom up tissue engineering approach. ${ }^{[42]}$

However, to create truly biomimetic microgels, the incorporation of biomimetic elements such as extracellular matrix components, growth factor binding sites, and cell responsive elements such as catabolically cleavable moieties will be of deciding importance. Advantageously, enzymatically crosslinked biomaterials such as Dex-TA allow for straightforward decoration with such elements. For example, cell instructing and growth factor capturing matrix molecules including hyaluronic acid, heparin, and chondroitin sulfate can be engrafted on the polymer's backbone or co-crosslinked using, e.g., tyramine modified molecules. ${ }^{[18,19,43]}$ Enzymatic crosslink strategies such as used for Dex-TA can also covalently bond a myriad of molecules including collagens by crosslinking with their tyrosine residues. ${ }^{[4]}$

In summary, enzymatically crosslinked hydrogels are ideal for the generation of microfluidically generated microgels. This platform is expected to contribute to the future development of custom-designed 3D biomimetic microtissues using a plethora of existing, simple and costeffective methods to enable cell-based screenings with a single cell resolution.

\section{Experimental Section}

\subsection{Microfluidic Chip Production}

Microfluidic devices were made using a standard soft lithography process. In short, microfluidic channels were designed using CAD software (Clewin, Wieweb software) and patterned onto a chromium photomask. A layer of SU-8 polymer $(70 \mu \mathrm{m})$ was spun on 
a silicon wafer and exposed to UV through the photomask, and was subsequently developed to produce the mold. Polydimethylsiloxane (PDMS, Sylgard 184, Dow Corning) was mixed in a 10:1 base:curing agent ratio, casted on the mold and cured. The PDMS was peeled from the mold, holes were punched to create inlets and outlets, and the PDMS was bound to a glass slide using oxygen plasma. A schematic representation, a microphotograph, and scanning electron images of key elements of the universal chip design are shown in Figure 1.

\subsection{Cell Culture}

The use of patient material was approved by the local ethical committee of the Medisch Spectrum Twente, and informed written consent was obtained for all samples. Human mesenchymal stem cells (hMSCs) were isolated from bone marrow as described previously. ${ }^{[45]}$ The cells were cultured in Alfa-MEM (Invitrogen), supplemented with heat-inactivated FBS (10\%, Lonza), L-glutamine ( $2 \times 10^{-3} \mathrm{M}$, Invitrogen), ascorbic acid $\left(0.2 \times 10^{-3} \mathrm{M}\right.$, Sigma-Aldrich),

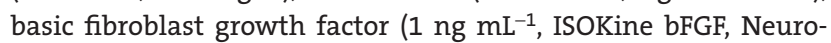
mics), penicillin (100 $\left.\mathrm{U} \mathrm{mL}^{-1}\right)$, and streptomycin $\left(100 \mu \mathrm{g} \mathrm{mL} \mathrm{m}^{-1}\right.$, Invitrogen). Cells were kept in a humidified environment with $5 \%$ $\mathrm{CO}_{2}$ and used from passage 3 to 5 .

\subsection{Microgel Formation}

Fluids were infused into chips using syringe pumps (Cetoni $\mathrm{GmbH}$ ). The continuous phase consisted of hexadecane (SigmaAldrich) with Span 80 surfactant (1\% (w/w), Sigma-Aldrich), PEGDA, alginate and Dex-TA were used as the dispersed phase. PEGDA (10 w/v\%, $3400 \mathrm{~g} \mathrm{~mol}^{-1}$, Lysan Bio) in PBS containing 2-hydroxy-4'-(2-hydroxyethoxy)-2-methylpropiophenone (0.2\%, Irgacure 2959, Sigma-Aldrich) was infused at a flow rate ratio of PEG:Oil 1.4:10 (water/oil ratio $=0.14$ ). Droplets were exposed to UV in the delay channel $\left(140 \mathrm{~mW} \mathrm{~cm}^{-2}\right.$, LC8 Lightningcure L9588, Hamamatsu), unless stated otherwise. This resulted in a gelation time of approximately $8 \mathrm{~s}$. Alginate ( $2 \%$, Sigma-Aldrich, low viscosity, 235 CPS) in PBS, supplemented with calcium carbonate nanoparticles (3.3 $\mathrm{mg} \mathrm{mL}^{-1}$, Nanomaterials Technology), was used at an alginate:oil flow rate ratio of 3:40 (water/oil ratio $=0.075$ ). Alginate was gelated by infusing of acetic acid containing hexadecane $(0.44-1.33 \mu \mathrm{L}$ acid per $\mathrm{mL}$ oil) in the delay channel. Dex-TA in PBS (10 w/v\%, DS $=15$, synthesized as described previously ${ }^{[22]}$ ) was coflown with HRP and $\mathrm{H}_{2} \mathrm{O}_{2}$ in DexTA. The flow rate ratio of Dex-TA:HRP: $\mathrm{H}_{2} \mathrm{O}_{2}$ :oil was respectively 6:1.2:1.2:60 (water/oil ratio $=0.14$ ). Final concentrations were HRP $0.057 \mathrm{mg} \mathrm{mL}^{-1}$ (250 units $\mathrm{mg}^{-1}$, Sigma-Aldrich) and $\mathrm{H}_{2} \mathrm{O}_{2}$ $15.75 \times 10^{-3}$ M (Sigma-Aldrich) unless stated otherwise. Microgels were collected in culture medium, which in the case of alginate was supplemented with $80 \times 10^{-3} \mathrm{M} \mathrm{CaCl}_{2}$ to ensure optimal crosslinking. When encapsulating cells, the hydrogel precursors were laden with $15 \times 10^{6} \mathrm{hMSCs}$ per $\mathrm{mL}$.

\subsection{Scanning Electron Microscopy}

Samples were dehydrated in graded ethanol series (50\%-100\%), dried from Hexamethyldisilazane (HMDS, Merck), gold sputtered (Cressington), and imaged (XL30 ESEM, FEI).

\subsection{Viability and Metabolic Activity}

Live/dead assay was performed using ethidium homodimer-1 and calcein-AM (Invitrogen) according to manufacturer's protocol at 2-4 $\mathrm{h}$ post-encapsulation, microphotographed (Nikon E600 fluorescence microscope), and quantified using ImageJ software ( $\mathrm{N}=30$ microgels). Metabolic activity was assessed at day 1, 4, 7, 14, 21, and 28 using the Presto Blue assay (Invitrogen) following manufacturer's protocol. Metabolic activities were normalized to day 1. Presto Blue assay results were corroborated with MTT staining at day 1 and 7 (Sigma-Aldrich) (Figure S1, Supporting Information). MTT was added to cell-laden microgels cultures at $0.5 \mathrm{mg} \mathrm{mL}$, which were microphotographed after $2 \mathrm{~h}$ of incubation and quantified using Image software ( $>15$ microgels).

\subsection{Confocal Microscopy}

Gels were fixated using formalin, cytoskeleton was stained with Phalloidin-AF488 (Invitrogen), and nuclei were stained with DRAQ5 (Invitrogen). Stained gels were imaged using confocal microscopy (Nikon A1 confocal microscope).

\subsection{Micromechanical Testing}

Micromechanical testing was done with a JPK Nanowizard AFM combined with Zeiss inverted optical microscope, using the Colloidal Probe technique. The cantilever spring constant was $0.151 \mathrm{~N} \mathrm{~m}^{-1}$ for all samples. Measurements were performed at ambient temperature in PBS, with exception of the alginate samples, which were supplemented with $80 \times 10^{-3} \mathrm{M} \mathrm{of} \mathrm{CaCl}_{2}$ to prevent ion loss.

\subsection{Statistical Analysis}

All experiments consisted of biological triplicates at minimum. All data were shown as average \pm standard deviation (SD), unless stated differently. Data were analyzed for statistical significance using ANOVA with Bonferroni post hoc test, or with the KruskalWallis test for non-normal distributed data, with a p-value of 0.05 .

\section{Supporting Information}

Supporting Information is available from the Wiley Online Library or from the author.

Acknowledgements: The authors gratefully thank Johan Bomer of the BIOS Lab-on-a-chip group of University of Twente for his expertise in micromolding, Tom Kamperman and Jan Hendriks of the Developmental BioEngineering group of University of Twente for their assistance with Matlab analysis. This research was supported by the Dutch Fund for Economic Reinforcement (FES) and the Diabetes Fund Netherlands, as part of the diabetes cell therapy initiative. Dr. Leijten acknowledges financial support from Innovative Research Incentives Scheme Veni \#14328 of the Netherlands Organization for Scientific Research (NWO).

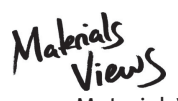

www.MaterialsViews.com 
Received: May 6, 2016; Revised: June 25, 2016; Published online: July 21, 2016; DOI: 10.1002/mabi.201600174

Keywords: biomaterials; enzymatic; hydrogel; microfluidic; microgels

[1] B. Ma, J. C. Leijten, L. Wu, M. Kip, C. A. van Blitterswijk, J. N. Post, M. Karperien, Osteoarthritis Cartilage 2013, 21, 599.

[2] P. Godoy, J. G. Hengstler, I. Ilkavets, C. Meyer, A. Bachmann, A. Müller, G. Tuschl, S. O. Mueller, S. Dooley, Hepatology 2009, 49, 2031.

[3] D.-A. Lacorre, E. S. Baekkevold, I. Garrido, P. Brandtzaeg, G. Haraldsen, F. Amalric, J.-P. Girard, Blood 2004, 103, 4164.

[4] J. Leijten, Y. C. Chai, I. Papantoniou, L. Geris, J. Schrooten, F. P. Luyten, Adv. Drug Delivery Rev. 2015, 84, 30.

[5] J. Leijten, J. Rouwkema, Y. S. Zhang, A. Nasajpour, M. R. Dokmeci, A. Khademhosseini, Small 2016, 12, 2130.

[6] J. Lee, M. J. Cuddihy, N. A. Kotov, Tissue Eng., Part B 2008, 14, 61.

[7] A. Kang, J. Park, J. Ju, G. S. Jeong, S.-H. Lee, Biomaterials 2014 35, 2651.

[8] P. de Vos, H. A. Lazarjani, D. Poncelet, M. M. Faas, Adv. Drug Delivery Rev. 2014, 67, 15.

[9] S. Allazetta, L. Kolb, S. Zerbib, J. a. Bardy, M. P. Lutolf, Small 2015, 11, 5647.

[10] C. J. Martinez, J. W. Kim, C. Ye, I. Ortiz, A. C. Rowat, M. Marquez, D. Weitz, Macromol. Biosci. 2012, 12, 946.

[11] E. Tumarkin, E. Kumacheva, Chem. Soc. Rev. 2009, 38 , 2161.

[12] E. Santos, J. Zarate, G. Orive, R. M. Hernandez, J. L. Pedraz, Adv. Exp. Med. Biol. 2010, 670, 5.

[13] Ý. A. Mørch, I. Donati, B. L. Strand, Biomacromolecules 2006, 7, 1471.

[14] Y. Ma, M. P. Neubauer, J. Thiele, A. Fery, W. T. S. Huck, Biomater. Sci. 2014, 2, 1661.

[15] C. Kielbassa, L. Roza, B. Epe, Carcinogenesis 1997, 18, 811.

[16] J. Clausell-Tormos, D. Lieber, J.-C. Baret, A. El-Harrak, O. J. Miller, L. Frenz, J. Blouwolff, K. J. Humphry, S. Köster, H. Duan, C. Holtze, D. A. Weitz, A. D. Griffiths, C. A. Merten, Chem. Biol. 2008, 15, 427.

[17] R. Jin, C. Hiemstra, Z. Zhong, J. Feijen, Biomaterials 2007, 28, 2791.

[18] J. W. H. Wennink, K. Niederer, A. I. Bochyńska, L. S. Moreira Teixeira, M. Karperien, J. Feijen, P. J. Dijkstra, Macromol. Symp. 2011, 309, 213.

[19] R. Jin, L. S. Moreira Teixeira, P. J. Dijkstra, C. A. van Blitterswijk, M. Karperien, J. Feijen, J. Controlled Release 2011, 152, 186.

[20] S. J. Bryant, K. S. Anseth, J. Biomed. Mater. Res. 2002, 59, 63.
[21] J. A. Nicell, H. Wright, Enzyme Microb. Technol. 1997, 21, 302.

[22] R. Jin, L. S. Moreira Teixeira, P. J. Dijkstra, Z. Zhong, C. A. van Blitterswijk, M. Karperien, J. Feijen, Tissue Eng., Part A 2010, 16, 2429.

[23] L. S. Moreira Teixeira, J. C. Leijten, J. Sobral, R. Jin, A. A. van Apeldoorn, J. Feijen, C. van Blitterswijk, P. J. Dijkstra, M. Karperien, Eur. Cell Mater. 2012, 23, 387.

[24] P. Panda, S. Ali, E. Lo, B. G. Chung, T. A. Hatton, A. Khademhosseini, P. S. Doyle, Lab Chip. 2008, 8, 1056.

[25] A. J. Engler, S. Sen, H. L. Sweeney, D. E. Discher, Cell 2006, 126,677

[26] C. G. Williams, T. K. Kim, A. Taboas, A. Malik, P. Manson, J. Elisseeff, Tissue Eng. 2003, 9, 679.

[27] G. Musumeci, C. Loreto, M. L. Carnazza, I. Strehin, J. Elisseeff, Histol. Histopathol. 2011, 26, 1265.

[28] W. H. Tan, S. Takeuchi, Adv. Mater. 2007, 19, 2696.

[29] M. Moya, M. Morley, O. Khanna, E. Opara, E. Brey, J. Mater. Sci. Mater. Med. 2012, 23, 903.

[30] L. I. Smith-Mungo, H. M. Kagan, Matrix Biol. 1998, 16, 387.

[31] D. Aeschlimann, O. Kaupp, M. Paulsson, J. Cell Biol. 1995, $129,881$.

[32] F. Passardi, C. Penel, C. Dunand, Trends Plant Sci. 2004, 9, 534.

[33] A. Buxboim, I. L. Ivanovska, D. E. Discher, J. Cell Sci. 2010, 123, 297.

[34] S. Guven, P. Chen, F. Inci, S. Tasoglu, B. Erkmen, U. Demirci, Trends Biotechnol. 2015, 33, 269.

[35] Y. Du, E. Lo, S. Ali, A. Khademhosseini, Proc. Natl. Acad. Sci. USA 2008, 105, 9522

[36] Y. T. Matsunaga, Y. Morimoto, S. Takeuchi, Adv. Mater. 2011, 23, $\mathrm{H} 90$.

[37] J. Leijten, A. Khademhosseini, Cell Stem Cell 2016, 18, 20.

[38] D. E. Discher, D. J. Mooney, P. W. Zandstra, Science 2009, 324, 1673.

[39] M.-Y. Lee, R. A. Kumar, S. M. Sukumaran, M. G. Hogg, D. S. Clark, J. S. Dordick, Proc. Natl. Acad. Sci. USA 2008, 105, 59.

[40] H. Onoe, T. Okitsu, A. Itou, M. Kato-Negishi, R. Gojo, D. Kiriya, K. Sato, S. Miura, S. Iwanaga, K. Kuribayashi-Shigetomi, Y. T. Matsunaga, Y. Shimoyama, S. Takeuchi, Nat. Mater. 2013, 12, 584

[41] S. Gobaa, S. Hoehnel, M. Roccio, A. Negro, S. Kobel, M. P. Lutolf, Nat. Methods 2011, 8, 949.

[42] A. Khademhosseini, R. Langer, Biomaterials 2007, $28,5087$.

[43] L. S. Moreira Teixeira, J. C. H. Leijten, J. W. H. Wennink, A. G. Chatterjea, J. Feijen, C. A. van Blitterswijk, P. J. Dijkstra, M. Karperien, Biomaterials 2012, 33, 3651.

[44] L. S. Moreira Teixeira, S. Bijl, V. V. Pully, C. Otto, R. Jin, J. Feijen, C. A. van Blitterswijk, P. J. Dijkstra, M. Karperien, Biomaterials 2012, 33, 3164.

[45] S. K. Both, A. J. van der Muijsenberg, C. A. van Blitterswijk, J. de Boer, J. D. de Bruijn, Tissue Eng. 2007, 13, 3. 